\title{
Desempenho de Crianças e Adolescentes Não Pacientes no Zulliger SC
}

\author{
Ana Clara Mateus Carvalho ${ }^{1}$, Ana Cristina Resende \\ Pontifícia Universidade Católica de Goiás, Goiânia-GO, Brasil
}

RESUMO

Este artigo apresenta e compara o desempenho de crianças no Zulliger Sistema Compreensivo, ZSC, de acordo com a idade. Assim, 304 crianças, de ambos os sexos, de 7 a 14 anos, que frequentavam escolas públicas e particulares, foram avaliadas pelo ZSC, considerando 70 variáveis do teste. A análise dos dados utilizou estatística descritiva, ANOVA One-Way (teste post hoc de Tukey), teste de $t$ Student e o teste $d$ Cohen. Os resultados mostraram diferenças significativas que permitiram dividir a amostra em três grupos. Onze variáveis mostraram diferenças significativas entre os grupos com tamanhos de efeito entre moderado e grande. O aspecto mais evidente foi a evolução cognitiva ao longo do desenvolvimento, corroborando com a literatura. Os dados levantados demonstraram que o ZSC é um instrumento que pode ser válido para avaliação psicológica da faixa etária estudada.

Palavras-chave: Zulliger SC, desempenho, crianças, adolescentes.

\section{ABSTRACT - Performance of Non-Patient Children and Adolescents in Zulliger SC}

This article presents and compares the performance of children in the Zulliger Comprehensive System (ZSC) according to age. A sample of 304 children of both sexes, from 7 to 14 years of age, attending public and private schools, were evaluated by ZSC, considering 70 variables of the test. Data analysis incorporated descriptive statistics, One-way ANOVA (Tukey post hoc test), $t$ Student test and Cohen's test. The results showed significant differences that allowed sample division into three groups. Eleven variables showed significant differences between groups with moderate to large effect sizes. The most evident aspect was the developmental cognitive evolution, corroborating findings in the literature. The data showed that ZSC is a valid instrument for psychological evaluation of the studied age group.

Keywords: Zulliger CS, performance, children, adolescents.

RESUMEN - El rendimiento de los Niños y Adolescentes no Pacientes en Zulliger SC

Este artículo presenta y compara el desempeño de niños en el Zulliger Sistema Comprensivo, ZSC, de acuerdo a la edad. Por lo tanto, 304 niños de ambos sexos, de 7 a 14 años, que frecuentan escuelas públicas y privadas fueron evaluados por ZSC, teniendo en cuenta 70 variables del test. El análisis de datos utilizó estadística descriptiva, ANOVA One-way (Tukey test post hoc), test de $t$ Student y el test de Cohen. Los resultados mostraron diferencias significativas que permitieron dividir la muestra en tres grupos. Once variables mostraron diferencias significativas entre los grupos de efectos moderados y los de gran tamaño. El aspecto más evidente fue la evolución cognitiva a lo largo del desarrollo, corroborando con la literatura. Los datos obtenidos mostraron que el ZSC es un instrumento válido para la evaluación psicológica del grupo de edad estudiado.

Palabras clave: Zulliger SC, desempeño, niños, adolescentes.

O Zulliger é um teste psicológico, constituído por três cartões com manchas de tinta, desenvolvidos à imagem e semelhança do Método de Rorschach que, por sua vez, é constituído por dez cartões. Ambos também têm o mesmo propósito, o de avaliar a personalidade, enquanto modo de pensar, sentir e agir. Devido à semelhança entre os dois instrumentos, Villemor-Amaral e Primi (2012) optaram por adaptar o Sistema Compreensivo (SC), já utilizado com sucesso no método de Rorschach (Exner, 2003; Meyer at al., 2002; Weiner \& Craighead, 2010), para a codificação e análise do Teste de Zulliger em adultos. No presente estudo, manteve-se o mesmo sistema de correção do Zulliger SC em adultos para crianças e adolescentes, uma vez que outros estudos têm demonstrado indícios de validade dessa prática em crianças e adolescentes de 7 a 14 anos, que é a faixa etária aqui abordada (Biasi \& VillemorAmaral, 2016; Villemor-Amaral, Pavan, Tavella, Cardoso, \& Biasi, 2016; Villemor-Amaral \& Vieira, 2016).

Contextualizando o período de 7 a 12 anos, de acordo com vários estudiosos (Coll, Marchesi, \& Palácios

${ }^{1}$ Endereço para correspondência: Rua C-165, Quadra 381, Lote 03, Setor Jardim América, 74275-170, Goiânia, GO. Tel.: (62) 9966-0812.

E-mail: anaclaramcarvalho@gmail.com

Apoio institucional: Fundação de Amparo à Pesquisa do Estado de Goiás (FAPEG). 
2008; Exner \& Weiner, 1995; Martí, 2008; Silva \& Campos, 2000), espera-se que as crianças estejam frequentando regularmente a escola e desenvolvendo amplamente suas competências no âmbito físico, cognitivo e psicossocial. Presume-se que essas crianças tenham recursos para planejar e utilizar suas habilidades para a resolução de problemas, que saibam discernir ideias relevantes de outras irrelevantes e que estejam desenvolvendo também a criatividade.

Outra faixa etária abarcada neste estudo é a de 13 e 14 anos, a primeira fase da adolescência, considerada uma etapa de transição no desenvolvimento humano. O desenvolvimento cognitivo, diferente nas crianças, tende a ser mais complexo. O raciocínio do adolescente que possui acesso à escolarização é propenso a ser mais preciso, independente, maduro e consolida-se justamente a partir dos 12 anos. Suas percepções acerca de realidade são mais precisas e de acordo com as demandas sociais (Borges, Matos \& Diniz, 2011; Carretero \& León, 2008; Ceballos, 2009; Inhelder \& Piaget, 1955; Pfundt \& Duit, 1993).

Nessa faixa etária, os jovens sentem mais necessidade de privacidade, aproximam-se dos amigos de forma mais intensa e afetiva, bem como o contexto interpessoal sofre mais influências do social e tudo isso contribui para a diminuição da interação com a família. Com a chegada das transformações e processos anátomo-fisiológicos da puberdade, há o aumento do impulso sexual e, eventualmente, uma queda da autoestima. É comum também um aumento do estresse situacional, gerando tristeza, angústia, retraimento, autocentramento e distanciamento de situações desagradáveis. Devido a esse processo, o adolescente adquire mais habilidade de perceber a si mesmo e o meio em que vive e passa a utilizar de recursos para neutralizar emoções dolorosas por intermédio da intelectualização (Campagna \& Souza, 2006; Carretero \& León, 2008; Eschenbeck, Kohlmann, \& Lohaus, 2007; Exner, 2003; Exner \& Sendin, 1999; Oliva, 2008; Poletto \& Koller, 2011; Segabinazi et al., 2012).

Nas últimas décadas, considerando especificamente os estudos com as manchas de tinta, tanto no método de Rorschach SC quanto no Zulliger SC, as questões de desenvolvimento psicológico em crianças e adolescentes também foram investigadas (Exner, 2003; Exner \& Weiner, 1995; Hosseininasab, Mohammadi, Weiner, \& Delavar, 2015; Leichtman, 1996; Meyer, Viglione, Mihura, Erard, \& Erdberg, 2007; Nascimento, Resende, \& Ribeiro, 2016; Resende, Carvalho, \& Martins, 2012; Ribeiro, Semer, \& Yazigi, 2011; Wenar \& Curtis, 1991). Esses estudos revelaram que quanto mais novas são as crianças menos recursos psicológicos eficientes elas têm para administrar situações de estresse no seu cotidiano; menos capazes são de antecipar as consequências de seus atos; mais simples, superficiais e estereotipadas são a percepção do mundo e das pessoas; mais reatividade emocional e impulsividade elas apresentam.
Assim, com o aumento da idade, também tende a aumentar a capacidade e a sofisticação dos processos cognitivos, bem como a capacidade de se envolver em relacionamentos interpessoais, para discriminar e melhor regular estados emocionais, para usar e incorporar as influências emocionais de uma forma mais madura e internalizar as preocupações (Biasi \& Villemor-Amaral, 2016; Villemor-Amaral et al., 2016; Villemor-Amaral \& Vieira, 2016).

Em referência aos estudos do ZSC, específicos para crianças e adolescentes, após um levantamento bibliográfico nas bases BVS-Psi, SciELO, PubMed, Medline, PsycNet, Web of Science, BDTD e repositórios de teses e dissertações das universidades USF, UFMG, UFU, UFRGS, PUC RS, UFSC, USP e UnB, foram encontradas cinco publicações que evidenciaram a validade do uso desse teste para essa faixa etária (Biasi \& Villemor-Amaral, 2016; Tavella \& Villemor-Amaral, 2014; Villemor-Amaral et al., 2016; Villemor-Amaral \& Quirino, 2013; Villemor-Amaral \& Vieira, 2016). Os estudos abarcaram evidências de validade do ZSC com crianças (Villemor-Amaral et al., 2016), relacionamento interpessoal (Biasi \& Villemor-Amaral, 2016), maturidade relacional (Villemor-Amaral \& Vieira, 2016), indicadores do potencial criativo em crianças (Tavella \& Villemor-Amaral, 2014) e indicadores afetivos e cognitivos (Villemor-Amaral \& Quirino, 2013). No entanto, nenhum estudo se dedicou ao levantamento de desempenhos padrões de crianças e adolescentes no ZSC. Diante disso, o presente estudo teve como objetivo geral contribuir com a construção de parâmetros psicométricos brasileiros de desempenho padrão de crianças e adolescentes no ZSC. O objetivo especifico consiste em investigar se há diferença entre as idades por grupos etários. Assim, este estudo caracteriza-se como empírico, descritivo, do tipo levantamento por amostragem, que procura estabelecer e comparar desempenhos padrões em um instrumento de avaliação psicológica em função da idade e sexo.

\section{Método}

\section{Participantes}

Inicialmente participaram desta pesquisa 324 crianças e adolescentes voluntários, com idades entre 7 e 14 anos, tanto do sexo masculino quanto feminino, provenientes de escolas públicas e particulares. Os critérios de inclusão dos participantes foram: ter o Termo de Consentimento Livre e Esclarecido (TCLE) assinado por um dos responsáveis, ter o formulário sobre as condições psicológicas e somáticas preenchido corretamente e cursar o ano ou série correspondente à sua faixa etária. Os critérios de exclusão foram: não ter como língua materna o português brasileiro, estar em tratamento psicológico ou psiquiátrico no último ano, apresentar indícios no formulário de algum distúrbio psicológico ou somático grave e apresentar um nível de inteligência geral não 
verbal indicando intelectualmente deficiente no Teste de Raven - Escala Geral ou Especial (percentil $\leq 5$ ).

De acordo com esses critérios, 20 participantes não puderam compor a amostra: seis $(1,9 \%)$ foram eliminados por indício de deficiência mental grave no Raven, dois deles $(0,6 \%)$ por não terem como língua materna o português brasileiro e $11(3,4 \%)$ por possuírem algum distúrbio psicológico ou somático, como constava no formulário de caracterização dos participantes preenchidos pelos responsáveis do participante. Ao final, compuseram a amostra do estudo 304 crianças e adolescentes não pacientes $(M=10,27$ anos e $D P=2,2)$. Como forma de representar as experiências culturais e sociais que devem ser retratadas em estudos que buscam o desempenho médio de crianças, foi utilizada a origem escolar (pública e particular) como um indicador dos diferentes cenários socioeconômico-culturais. As escolas participantes da pesquisa foram escolhidas de acordo com critérios de conveniência. A Tabela 1 apresenta aspectos sociodemográficos e econômicos dos participantes deste estudo.

Tabela 1

Dados Demográficos dos Três Grupos de Participantes da Amostra Normativa do Teste de Zulliger SC

\begin{tabular}{|c|c|c|c|}
\hline Variáveis & Grupo $1(N=115)$ & Grupo $2(N=128)$ & Grupo $3(N=61)$ \\
\hline \multicolumn{4}{|l|}{ Sexo } \\
\hline feminino & $61(53 \%)$ & $59(46 \%)$ & $36(59 \%)$ \\
\hline masculino & $54(47 \%)$ & $69(54 \%)$ & $25(41 \%)$ \\
\hline \multicolumn{4}{|l|}{ Tipo de Escola } \\
\hline particular & $74(64,3 \%)$ & $61(47,6 \%)$ & $41(67,2 \%)$ \\
\hline pública & $41(35,6 \%)$ & $66(52 \%)$ & $20(32,7 \%)$ \\
\hline \multicolumn{4}{|l|}{ Nível Socioeconômico* } \\
\hline $\mathrm{D}$ & $5(4,3 \%)$ & $0(0 \%)$ & $0(0 \%)$ \\
\hline $\mathrm{C}$ & $21(18,2 \%)$ & $23(17,9 \%)$ & $3(5 \%)$ \\
\hline B & $61(53 \%)$ & $66(52 \%)$ & $39(63,9 \%)$ \\
\hline A & $14(12 \%)$ & $25(20 \%)$ & $17(27,8 \%)$ \\
\hline Total & $101(87,8 \%)$ & $114(89 \%)$ & $59(96,7 \%)$ \\
\hline
\end{tabular}

Nota. *Renda média bruta familiar mensal [item respondido por apenas 274 pessoas, 90,1\% da amostra, ou seja, 30 pessoas não responderam (9,9\%)]: nível D: R\$ 895,00; nível C: R\$ 1.865,00; nível B: R\$ 3.118,00; nível A: R\$ 11.037,00

Este estudo possui, em sua maioria, participantes do sexo feminino (51,3\%), de escola particular $(57,9 \%)$. O nível socioeconômico de renda, informado pelos pais, foi médio (nível B), o que equivale a uma renda aproximada de R \$ 3.118,00 (Três mil, cento e dezoito reais), conforme os critérios estabelecidos pela Associação Brasileira de Empresas de Pesquisa (ABEP, 2014). Os participantes foram divididos em três grupos etários: o Grupo 1, com crianças de 7 a 9 anos; Grupo 2, de 10 a 12 anos; Grupo 3 composto por adolescentes de 13 e 14 anos.

\section{Instrumentos}

Formulário de Caracterização dos Participantes. Esse instrumento foi utilizado para coletar informações a respeito do desenvolvimento psicomotor, social, emocional, assim como da linguagem e saúde dos participantes, com o objetivo de identificar algum indício de distúrbio psicológico ou somático grave (critério de exclusão do presente estudo). Por meio desse instrumento também levantou-se informações sobre sexo, idade, escolaridade e dados socioeconômicos.

Teste Matrizes Progressivas de Raven Escalas Especial e Geral. Esse instrumento tem como objetivo avaliar a inteligência geral não verbal, o fator g, que é a capacidade de observar, pensar e verificar o nível de desenvolvimento intelectual. A Escala Especial (Angelini, Alves, Custódio, Duarte, \& Duarte, 1999), destinada a crianças de 5 a 11 anos e oito meses, foi administrada nos participantes de 7 a 12 anos e 6 meses, devido à proximidade entre a idade de 12 anos e a idade limite apresentadas nas normas do manual. A Escala Geral (Raven, 2008) é semelhante à Escala Especial, porém destinada a adolescentes, a partir de 13 anos. É constituída por itens que se tornam progressivamente mais complexos e que são respondidos sob a forma de múltipla escolha. Neste estudo, esse instrumento foi aplicado aos participantes acima de 12 anos e 6 meses, devido à proximidade da idade limite.

Teste de Zulliger no Sistema Compreensivo ZSC - Forma Individual. Avalia a estrutura e a dinâmica da personalidade, indicando as dificuldades, conflitos e as habilidades que constituem os recursos psíquicos que uma pessoa dispõe para lidar com os problemas. Também é um instrumento que pode ser aplicado em qualquer indivíduo, de qualquer nível socioeconômico-cultural, desde que tenha condições de se expressar verbalmente e que tenha suficiente acuidade visual. O teste é composto por um jogo de três 
cartões que contém uma mancha de tinta simétrica e diferente para cada um deles, sendo uma acromática, uma policromática e outra em preto e vermelho. A aplicação do teste consiste em mostrar uma lâmina de cada vez e pedir que a pessoa diga "o que isto poderia ser". Após anotar as respostas dadas aos três cartões, estes são repassados, realizando-se um inquérito com a finalidade de verificar "onde foi que a pessoa viu" e "o que, na mancha, fez com que parecesse aquilo" que foi dito. As respostas são classificadas e codificadas em diversas categorias, de acordo com os pressupostos do Sistema Compreensivo de Exner (Exner, 2003; Villemor-Amaral \& Primi, 2012). Ao total foram analisadas 70 variáveis do teste.

\section{Procedimentos}

Este estudo faz parte de um projeto de pesquisa maior, aprovado pelo Comitê de Ética em Pesquisa da Pesquisa da Pontifícia Universidade Católica de Goiás, sob o CAAE $n^{\circ}$ 0156.0.168.000-11 e recebeu o apoio financeiro da Fundação de Amparo à Pesquisa do Estado de Goiás (FAPEG). Inicialmente, foi realizada a capacitação da equipe pesquisadora para a coleta dos dados mediante treinamentos sistematizados. Essa equipe foi composta por cinco pesquisadoras psicólogas, que tinham, no mínimo, 130 horas de treino na aplicação e correção do Sistema Compreensivo.

O segundo passo foi o contato com escolas particulares e as Secretarias Municipal e Estadual de Educação, que autorizaram a realização da pesquisa em escolas oriundas de diferentes regiões da capital, além de escolas de outros municípios do estado. Ao total, seis escolas particulares e seis escolas públicas participaram como voluntárias na capital, além de outras quatro escolas do interior do estado, sendo duas particulares e duas públicas. Posteriormente, os TCLE e os Formulários de identificação dos participantes foram entregues aos pais ou aos responsáveis pelos alunos dessas escolas. Todos os alunos que tiveram os seus termos de consentimento assinados e os formulários preenchidos corretamente participaram do estudo.

As examinadoras, sempre em duplas, administravam o Teste de Raven em pequenos grupos de aproximadamente 10 participantes, em encontros que variavam entre 30 e 45 minutos e, sempre em duplas, corrigiam logo em seguida o material, segundo os manuais desse instrumento. Em outro dia, as examinadoras administravam individualmente o ZSC, que tinha a duração entre 15 e 30 minutos. Os protocolos do ZSC foram codificados pela responsável pela aplicação e revisados pela primeira autora deste artigo.

\section{Análise da Fidedignidade entre Juízes}

Investigou-se a fidedignidade entre avaliadores acerca das codificações de variáveis do ZSC para $24 \%$ dos protocolos $(N=73)$, que foram selecionados aleatoriamente e codificados por um juiz cego às codificações iniciais. Para o cálculo de concordância entre juízes, utilizou-se o kappa de Cohen. Os níveis de concordância entre juízes das principais variáveis do ZSC, que variaram de 0,70 a 0,91 [Localizações (4 variáveis) $=0,91$; Qualidade Evolutiva (4 variáveis) $=0,75$; Determinantes (11 variáveis) $=0,81$; Qualidade Formal (5 variáveis) $=0,70$; Par $=0,09$; Conteúdos (27 variáveis $=0,90$; Popular $=0,74$; Frequência de Nota $\mathrm{Z}=0,84$; Códigos Especiais (14 variáveis) $=0,82]$. Esses níveis de concordância podem ser considerados moderados $(0,40$ a 0,75$)$ e excelentes (acima de 0,75$)$, segundo Fleiss (1981). Esses resultados indicaram que a codificação dos protocolos do ZSC pode ser considerada confiável. Os protocolos que apresentaram divergências na codificação foram discutidos, resultando em uma codificação mais adequada, conforme acordo entre os juízes.

\section{Análise dos Dados}

O estudo seguiu todos os dispositivos da Resolução 466/12 do Conselho Nacional de Saúde e seus complementos. As variáveis de todos os protocolos foram lançadas no Statistical Package for the Social Sciences 18.0 (SPSS 18.0). A primeira etapa de análise dos resultados consistiu na verificação de possíveis diferenças entre as idades por meio da one-way ANOVA (post hoc Tukey), considerando as 70 variáveis selecionadas para este estudo. Esse teste estatístico permitiu dividir a amostra em três grupos etários (G1, G2 e G3), de modo a garantir que não existiam diferenças significativas entre as crianças inseridas em um mesmo grupo. Em seguida, os dados foram tratados por meio de estatística descritiva para a elaboração das tabelas de desempenho de cada grupo no teste por meio dos seguintes indicadores: média, desvio padrão, mínimo, máximo, percentil 25 , percentil 50 , percentil 75 , assimetria e curtose.

Posteriormente, foram realizadas análises comparativas entre os três grupos etários por meio da one-way ANOVA, trabalhando com o nível de significância menor ou igual a $0,05(p \leq 0,05)$. O teste $t$ Student também foi utilizado, com a probabilidade de erro menor ou igual a $0,05(p \leq 0,05)$, a fim de verificar se havia diferenças significativas entre os sexos, Junto às análises comparativas estimou-se a magnitude do efeito pelo $d$ de Cohen, considerando médios os valores entre 0,30 e 0,49, e grandes aqueles acima de 0,49 (Cohen, 1988).

\section{Resultados e Discussão}

Ao total foram identificadas oito variáveis do ZSC (11,4\% das 70 variáveis estudadas) que revelaram diferenças estatisticamente significativas entre os grupos etários, com grande magnitude de efeito para quatro 
destas $(7,1 \%)$, demonstrando desempenhos típicos para os grupos etários. As comparações entre os três grupos, considerando essas oito variáveis, por meio da ANOVA e do $d$ de Cohen são apresentadas na Tabela 2.

Tabela 2

Comparações entre os Grupos Etários por meio da ANOVA e d de Cohen

\begin{tabular}{|c|c|c|c|c|c|c|c|}
\hline Comparações & Variáveis & Grupo & $M$ & $\mathrm{DP}$ & $F$ & $p$ & $d$ \\
\hline \multirow{2}{*}{ G1 e G2 } & \multirow{2}{*}{ INC } & G1 & 0,33 & 0,72 & \multirow{2}{*}{0,20} & \multirow{2}{*}{0,00} & \multirow{2}{*}{0,36} \\
\hline & & G2 & 0,13 & 0,39 & & & \\
\hline \multirow{16}{*}{ G1 e G3 } & \multirow{2}{*}{ Ls } & G1 & 0,03 & 0,18 & \multirow{2}{*}{$-0,11$} & \multirow{2}{*}{0,03} & \multirow{2}{*}{$-0,46$} \\
\hline & & G3 & 0,15 & 0,35 & & & \\
\hline & \multirow{2}{*}{$\mathrm{Cg}$} & G1 & 0,43 & 0,63 & \multirow{2}{*}{0,24} & \multirow{2}{*}{0,03} & \multirow{2}{*}{0,46} \\
\hline & & G3 & 0,18 & 0,46 & & & \\
\hline & \multirow{2}{*}{ DQv } & G1 & 0,11 & 0,49 & \multirow{2}{*}{1,13} & \multirow{2}{*}{0,00} & \multirow{2}{*}{0,45} \\
\hline & & G3 & 0,00 & 0,00 & & & \\
\hline & \multirow{2}{*}{ FQnone } & G1 & 0,29 & 0,23 & \multirow{2}{*}{0,12} & \multirow{2}{*}{0,04} & \multirow{2}{*}{0,56} \\
\hline & & G3 & 0,00 & 0,00 & & & \\
\hline & \multirow{2}{*}{ INC } & G1 & 0,33 & 0,72 & \multirow{2}{*}{0,24} & \multirow{2}{*}{0,01} & \multirow{2}{*}{0,48} \\
\hline & & G3 & 0,08 & 0,33 & & & \\
\hline & \multirow{2}{*}{ FAB } & G1 & 0,14 & 0,60 & \multirow{2}{*}{0,13} & \multirow{2}{*}{0,00} & \multirow{2}{*}{0,46} \\
\hline & & G3 & 0,00 & 0,00 & & & \\
\hline & \multirow{2}{*}{ Sum6 } & G1 & 0,70 & 1,19 & \multirow{2}{*}{0,15} & \multirow{2}{*}{0,04} & \\
\hline & & G3 & 0,31 & 0,62 & & & 0,43 \\
\hline & & G1 & 1,84 & 3,56 & & & \\
\hline & WSum6 & G3 & 0,55 & 1,27 & 1,28 & 0,01 & 0,53 \\
\hline & DOv & G2 & 0,17 & 0,18 & 0.07 & 000 & 056 \\
\hline & $D<v$ & G3 & 0,00 & 0,00 & & 0,00 & 0,30 \\
\hline G2 e G3 & & & & & & & \\
\hline & FAB & G2 & 0,19 & 0,23 & 0,09 & 0,00 & 0,50 \\
\hline & & G3 & 0,00 & 0,00 & & & \\
\hline
\end{tabular}

Ao comparar os grupos G1 e G2, observou-se que o desempenho das crianças deste estudo foi muito semelhante, independentemente do grupo etário. Uma única variável (INC) revelou que as crianças com menos idade (G1) tendem a expressar o seu pensamento de uma forma mais imatura e fantasiosa do que as crianças com mais idade. Isso seria esperado, uma vez que, à medida que a idade aumenta, a criança se torna mais consciente de seus processos cognitivos, o que aumenta sua percepção e a torna mais capaz de avaliar o que pensa e como pensa (Martí, 2008). Esse aspecto foi observado pela frequência significativamente maior da variável INC em crianças mais jovens que no grupo de crianças com mais idade, bem como por meio de um tamanho de efeito moderado dessa variável entre os dois grupos $\left(\mathrm{INC}_{\mathrm{G} 1} M=0,33 ; D P=0,72\right.$; $\left.d=0,36 ; \mathrm{INC}_{\mathrm{G} 2} M=0,13 ; D P=0,39 ; d=0,36\right)$.

Considerando as crianças com menos idade (G1) e os adolescentes (G3), observou-se que à variável Ls foi mais frequente, com tamanho de efeito moderado, no grupo de adolescentes. Essa variável tem implicações no relacionamento interpessoal, o que sugere que os adolescentes utilizam frequentemente mais mecanismos para aliviar os conflitos afetivos, visando a sua melhor adequação à realidade, do que as crianças do G1. A elevada frequência do conteúdo paisagem (Ls) está relacionada à necessidade de distanciamento de situações conflituosas (Exner, 2003) e isso parece ser um mecanismo natural em alguns adolescentes, que buscam envolver-se mais em seus estudos do que em seus relacionamentos interpessoais (Carretero \& León, 2008).

Por outro lado, as crianças (G1) revelaram uma frequência significativamente maior da variável $\mathrm{Cg}$, com tamanho do efeito moderado, o que sugere maior necessidade de proteção e valorização da aparência $\left(\mathrm{C}_{\mathrm{gG} 1} M=0,43 ; D P=0,63 ; d=0,46\right)$. Nos primeiros anos do ensino fundamental, é comum, conforme Moreno 
(2008), que as crianças façam alusões a estereótipos e dividam-se em grupos que valorizem certas características, como aparência física ou rendimento acadêmico, para adquirir status. O estudo de Biasi (2013) corrobora essa teoria, pois avalia o relacionamento interpessoal de dois grupos de crianças do ensino fundamental, divididos em crianças populares e crianças rejeitadas, confirmando que, nessa fase, esse comportamento é habitual.

Em relação aos aspectos cognitivos, as crianças do G1 apresentaram uma frequência maior de uma série de variáveis (DQv, FQnone FAB, INC, Sum6 e WSum6), com tamanho do efeito variando entre moderado e grande (entre 0,43 e 0,53 ), que implicam em um funcionamento cognitivo menos sofisticado e mais imaturo do que aqueles presentes nos adolescentes (G3). Ou seja, as percepções das crianças revelaram-se mais desorganizadas, com falhas mais frequentes no processo de mediação cognitiva do que nos adolescentes, demonstrando que as crianças possuem menos capacidade de antecipar as consequências de seus atos e entender quais seriam os comportamentos mais adequados na maioria das situações. Assim, como seria esperado, os adolescentes apresentaram um fluxo de ideias e pensamentos mais compreensíveis do que as crianças mais novas, bem como revelaram-se mais capazes de organizar a informação, formular hipóteses e estabelecer uma relação de causa e efeito de uma dada situação, similar ao pensamento de um adulto. Nesse sentido, Inhelder e Piaget (1955) consideram que essa capacidade se dá pelo fato de as operações formais se consolidarem por volta da faixa etária de 13 e 14 anos, em que o raciocínio do adolescente atinge um nível mais complexo.

Nas últimas décadas, vários estudos com as manchas de tinta já constataram essas diferenças entre crianças e adolescentes no âmbito cognitivo, ou seja, quanto mais jovem é o indivíduo mais propenso a organizar as informações de modo ineficaz. Assim, com o aumento da idade, também tende a aumentar a capacidade e a sofisticação dos processos cognitivos (Exner, 2003; Exner \& Weiner, 1995; Leichtman, 1996; Meyer, Viglione, Mihura, Erard, \& Erdberg, 2007; Nascimento, Resende, \& Ribeiro, 2016; Resende, Carvalho \& Martins, 2012; Ribeiro, Semer, \& Yazigi, 2011, Villemor-Amaral et al., 2016; VillemorAmaral \& Vieira, 2016; Wenar \& Curtis, 1991 ).

No que diz respeito aos grupos de crianças com mais idade (G2) e adolescentes (G3), observou-se mais uma vez que os adolescentes possuem percepções mais precisas, menos fantasiosas e mais de acordo com as demandas e expectativas sociais, em comparação às crianças do G2, cujas percepções tendem a ser mais imprecisas e confusas da realidade. Esses aspectos foram observados por meio das variáveis $\mathrm{FAB}$ e DQv, que foram significativamente maiores nas crianças do que nos adolescentes, com tamanho do efeito grande para as duas. Esses dados estatísticos corroboram os estudos dos autores citados (Villemor-Amaral et al. 2016; Villemor-Amaral \& Vieira, 2016). Ainda que as crianças da faixa etária de 10 a 12 anos tenham desenvolvido a atenção seletiva, a memória estratégica e tenham o processamento da informação relativamente rápido, quando comparadas aos adolescentes, apresentam mais dificuldades em compreender e seguir demandas sociais, justamente por ainda possuírem um pensamento pueril, porém dentro do esperado para a sua fase do desenvolvimento (Martí, 2008).

Os dados referentes ao desempenho das crianças do G1, G2 e G3 no ZSC são expostos na Tabela 3, 4 e 5 respectivamente, com indicação da média, mediana, desvio padrão, dos valores mínimo e máximo, percentil 25 , percentil 50 , percentil 75 , assimetria e curtose.

Tabela 3

Estatísticas Descritivas de 115 Crianças de 7 a 9 Anos do G1 no ZSC

\begin{tabular}{lcccccccccc}
\hline \multicolumn{1}{c}{ Variável } & $M$ & Me & DP & Min & Max & Per 25 & Per 50 & Per 75 & Assim & Kurt \\
\hline R & 7,11 & 7,00 & 1,74 & 3,00 & 12,0 & 6,00 & 7,00 & 8,00 & 0,55 & 0,54 \\
W & 1,42 & 1,00 & 1,49 & 0,00 & 9,00 & 0,00 & 1,00 & 2,00 & 1,83 & 5,37 \\
D & 4,47 & 5,00 & 2,01 & 0,00 & 9,00 & 3,00 & 5,00 & 6,00 & $-0,17$ & $-0,23$ \\
Dd & 1,28 & 1,00 & 1,34 & 0,00 & 5,00 & 0,00 & 1,00 & 2,00 & 1,03 & 0,28 \\
S & 1,08 & 1,00 & 0,92 & 0,00 & 4,00 & 0,00 & 1,00 & 2,00 & 0,57 & $-0,15$ \\
DQ+ & 4,09 & 4,00 & 2,41 & 0,00 & 11,0 & 2,00 & 4,00 & 6,00 & 0,46 & 0,14 \\
DQo & 6,07 & 6,00 & 2,09 & 1,00 & 12,0 & 5,00 & 6,00 & 7,00 & 0,70 & 0,57 \\
DQv/+ & 0,01 & 0,00 & 0,09 & 0,00 & 1,00 & 0,00 & 0,00 & 0,00 & 10,72 & 115 \\
DQv & 0,11 & 0,00 & 0,49 & 0,00 & 4,00 & 0,00 & 0,00 & 0,00 & 5,67 & 37,6 \\
F & 4,50 & 4,00 & 2,26 & 0,00 & 11,0 & 3,00 & 4,00 & 6,00 & 0,53 & 0,21 \\
M & 0,63 & 0,00 & 0,95 & 0,00 & 4,00 & 0,00 & 0,00 & 1,00 & 1,70 & 2,75 \\
FM & 0,41 & 0,00 & 0,70 & 0,00 & 3,00 & 0,00 & 0,00 & 1,00 & 1,74 & 2,58 \\
M & 0,22 & 0,00 & 0,52 & 0,00 & 3,00 & 0,00 & 0,00 & 0,00 & 2,77 & 8,55 \\
Ativo & 0,73 & 0,00 & 1,07 & 0,00 & 5,00 & 0,00 & 0,00 & 1,00 & 1,69 & 2,80 \\
Passivo & 0,56 & 0,00 & 1,05 & 0,00 & 6,00 & 0,00 & 0,00 & 1,00 & 2,94 & 11,3 \\
FC & 0,50 & 0,00 & 0,76 & 0,00 & 4,00 & 0,00 & 0,00 & 1,00 & 1,83 & 4,16
\end{tabular}


Tabela 3 (continuação)

Estatísticas Descritivas de 115 Crianças de 7 a 9 Anos do G1 no ZSC

\begin{tabular}{|c|c|c|c|c|c|c|c|c|c|c|}
\hline Variável & $M$ & $\mathrm{Me}$ & $D P$ & Min & $\operatorname{Max}$ & Per 25 & Per 50 & Per 75 & Assim & Kurt \\
\hline CF & 0,32 & 0,00 & 0,62 & 0,00 & 3,00 & 0,00 & 0,00 & 0,00 & 1,99 & 3,54 \\
\hline C & 0,09 & 0,00 & 0,31 & 0,00 & 2,00 & 0,00 & 0,00 & 0,00 & 3,81 & 15,3 \\
\hline FD & 0,11 & 0,00 & 0,34 & 0,00 & 2,00 & 0,00 & 0,00 & 0,00 & 3,11 & 9,72 \\
\hline SumC' & 0,52 & 0,00 & 0,89 & 0,00 & 5,00 & 0,00 & 0,00 & 0,00 & 2,38 & 7,10 \\
\hline SumV & 0,11 & 0,00 & 0,34 & 0,00 & 2,00 & 0,00 & 0,00 & 0,00 & 3,11 & 9,72 \\
\hline SumT & 0,80 & 0,00 & 0,30 & 0,00 & 2,00 & 0,00 & 0,00 & 0,00 & 4,12 & 18,1 \\
\hline SumY & 0,29 & 0,00 & 0,54 & 0,00 & 2,00 & 0,00 & 0,00 & 0,00 & 1,75 & 2,22 \\
\hline $\mathrm{Fr}+\mathrm{rF}$ & 0,03 & 0,00 & 0,20 & 0,00 & 2,00 & 0,00 & 0,00 & 0,00 & 8,57 & 76,6 \\
\hline GHR & 1,12 & 1,00 & 0,96 & 0,00 & 4,00 & 0,00 & 1,00 & 2,00 & 0,40 & $-0,58$ \\
\hline PHR & 0,83 & 1,00 & 1,06 & 0,00 & 5,00 & 0,00 & 1,00 & 1,00 & 1,48 & 2,25 \\
\hline (2) & 2,89 & 3,00 & 1,65 & 0,00 & 7,00 & 2,00 & 3,00 & 4,00 & 0,27 & 0,11 \\
\hline $\mathrm{FQ}+$ & 0,01 & 0,00 & 0,93 & 0,00 & 1,00 & 0,00 & 0,00 & 0,00 & 10,7 & 115 \\
\hline FQo & 3,09 & 3,00 & 1,57 & 0,00 & 7,00 & 2,00 & 3,00 & 4,00 & 0,26 & $-0,32$ \\
\hline FQu & 2,93 & 3,00 & 1,52 & 0,00 & 9,00 & 2,00 & 3,00 & 4,00 & 0,54 & 1,30 \\
\hline FQ- & 1,04 & 1,00 & 1,23 & 0,00 & 8,00 & 0,00 & 1,00 & 2,00 & 2,21 & 8,68 \\
\hline FQnone & 0,09 & 0,00 & 0,33 & 0,00 & 2,00 & 0,00 & 0,00 & 0,00 & 4,22 & 18,4 \\
\hline $\mathrm{H}$ & 0,95 & 1,00 & 0,95 & 0,00 & 4,00 & 0,00 & 1,00 & 2,00 & 0,90 & 0,54 \\
\hline $\mathrm{H}+(\mathrm{H})+\mathrm{Hd}+(\mathrm{Hd})$ & 1,83 & 2,00 & 1,35 & 0,00 & 7,00 & 1,00 & 2,00 & 3,00 & 0,73 & 0,94 \\
\hline A & 3,45 & 3,00 & 1,81 & 0,00 & 10,0 & 2,00 & 3,00 & 5,00 & 0,61 & 0,91 \\
\hline$A+(A)+A d+(A d)$ & 3,86 & 4,00 & 1,79 & 0,00 & 10,0 & 3,00 & 4,00 & 5,00 & 0,36 & 0,52 \\
\hline Art & 0,04 & 0,00 & 0,24 & 0,00 & 2,00 & 0,00 & 0,00 & 0,00 & 6,21 & 41,7 \\
\hline Ay & 0,09 & 0,00 & 0,31 & 0,00 & 2,00 & 0,00 & 0,00 & 0,00 & 3,81 & 15,3 \\
\hline $\mathrm{Na}$ & 0,12 & 0,00 & 0,00 & 0,00 & 3,00 & 0,00 & 0,00 & 0,00 & 4,32 & 22,1 \\
\hline $\mathrm{Cl}$ & 0,00 & 0,00 & 0,00 & 0,00 & 0,00 & 0,00 & 0,00 & 0,00 & - & - \\
\hline Bt & 0,65 & 0,00 & 0,85 & 0,00 & 3,00 & 0,00 & 0,00 & 1,00 & 1,07 & 0,14 \\
\hline Ls & 0,03 & 0,00 & 0,18 & 0,00 & 1,00 & 0,00 & 0,00 & 0,00 & 5,14 & 24,9 \\
\hline $\mathrm{Ge}$ & 0,00 & 0,00 & 0,00 & 0,00 & 0,00 & 0,00 & 0,00 & 0,00 & - & - \\
\hline Food & 0,09 & 0,00 & 0,33 & 0,00 & 2,00 & 0,00 & 0,00 & 0,00 & 4,22 & 18,4 \\
\hline $\mathrm{Bl}$ & 0,14 & 0,00 & 0,39 & 0,00 & 2,00 & 0,00 & 0,00 & 0,00 & 2,94 & 8,56 \\
\hline Sx & 0,00 & 0,00 & 0,00 & 0,00 & 0,00 & 0,00 & 0,00 & 0,00 & - & - \\
\hline $\mathrm{Fi}$ & 0,09 & 0,00 & 0,28 & 0,00 & 1,00 & 0,00 & 0,00 & 0,00 & 2,97 & 6,94 \\
\hline Ex & 0,02 & 0,00 & 0,13 & 0,00 & 1,00 & 0,00 & 0,00 & 1,00 & 7,48 & 54,9 \\
\hline $\mathrm{Cg}$ & 0,45 & 0,00 & 0,63 & 0,00 & 3,00 & 0,00 & 0,00 & 0,00 & 1,42 & 1,82 \\
\hline $\mathrm{P}$ & 1,34 & 1,00 & 0,96 & 0,00 & 4,00 & 1,00 & 1,00 & 2,00 & 0,16 & $-0,65$ \\
\hline DV & 0,08 & 0,00 & 0,27 & 0,00 & 1,00 & 0,00 & 0,00 & 0,00 & 3,18 & 8,27 \\
\hline INC & 0,33 & 0,00 & 0,72 & 0,00 & 4,00 & 0,00 & 0,00 & 0,00 & 2,67 & 8,02 \\
\hline DR & 0,11 & 0,00 & 0,49 & 0,00 & 4,00 & 0,00 & 0,00 & 0,00 & 5,67 & 37,6 \\
\hline FAB & 0,14 & 0,00 & 0,60 & 0,00 & 5,00 & 0,00 & 0,00 & 0,00 & 5,96 & 41,0 \\
\hline ALOG & 0,02 & 0,00 & 0,13 & 0,00 & 1,00 & 0,00 & 0,00 & 0,00 & 7,48 & 54,9 \\
\hline CONT & 0,02 & 0,00 & 0,18 & 0,00 & 2,00 & 0,00 & 0,00 & 0,00 & 10,7 & 115 \\
\hline Sum6 & 0,70 & 0,00 & 0,19 & 0,00 & 6,00 & 0,00 & 0,00 & 1,00 & 2,28 & 5,66 \\
\hline WSum6 & 1,84 & 0,00 & 3,56 & 0,00 & 20,0 & 0,00 & 0,00 & 2,00 & 2,97 & 10,2 \\
\hline WSumC & 0,71 & 0,50 & 1,00 & 0,00 & 5,00 & 0,00 & 0,50 & 1,00 & 1,80 & 3,38 \\
\hline EA & 1,33 & 1,00 & 1,46 & 0,00 & 6,50 & 0,00 & 1,00 & 2,00 & 1,42 & 1,76 \\
\hline Es & 1,63 & 1,00 & 1,71 & 0,00 & 10,0 & 0,00 & 1,00 & 2,00 & 1,68 & 4,35 \\
\hline SumC'-WSumC & $-0,16$ & 0,00 & 1,04 & $-4,00$ & 3,50 & $-0,50$ & 0,00 & 0,00 & $-0,77$ & 3,36 \\
\hline Afr & 0,56 & 0,50 & 0,22 & 0,25 & 1,70 & 0,42 & 0,50 & 0,66 & 1,60 & 4,34 \\
\hline Blends & 0,55 & 0,00 & 0,92 & 0,00 & 4,00 & 0,00 & 0,00 & 1,00 & 1,85 & 3,12 \\
\hline AG & 0,30 & 0,00 & 0,68 & 0,00 & 4,00 & 0,00 & 0,00 & 0,00 & 2,66 & 7,98 \\
\hline $\mathrm{COP}$ & 0,12 & 0,00 & 0,35 & 0,00 & 2,00 & 0,00 & 0,00 & 0,00 & 2,92 & 8,44 \\
\hline
\end{tabular}


Tabela 3 (continuação)

Estatísticas Descritivas de 115 Crianças de 7 a 9 Anos do G1 no ZSC

\begin{tabular}{lcccccccccc}
\hline \multicolumn{1}{c}{ Variável } & $M$ & Me & DP & Min & Max & Per 25 & Per 50 & Per 75 & Assim & Kurt \\
\hline MOR & 0,33 & 0,00 & 0,71 & 0,00 & 5,00 & 0,00 & 0,00 & 1,00 & 3,46 & 17,1 \\
PER & 0,10 & 0,00 & 0,40 & 0,00 & 3,00 & 0,00 & 0,00 & 0,00 & 4,81 & 26,9 \\
Isol & 0,95 & 1,00 & 1,18 & 0,00 & 6,00 & 0,00 & 1,00 & 2,00 & 1,39 & 2,16 \\
Isol/R & 0,13 & 0,09 & 0,17 & 0,00 & 1,00 & 0,00 & 0,09 & 0,22 & 1,80 & 5,05 \\
Intelec & 0,19 & 0,00 & 0,51 & 0,00 & 2,00 & 0,00 & 0,00 & 0,00 & 2,67 & 6,21 \\
XA\% & 0,84 & 0,86 & 0,15 & 0,20 & 1,00 & 0,75 & 0,86 & 1,00 & $-1,16$ & 2,09 \\
WDA\% & 0,97 & 1,00 & 0,07 & 0,50 & 1,00 & 1,00 & 1,00 & 1,00 & $-4,40$ & 20,0 \\
X+\% & 0,42 & 0,44 & 0,20 & 0,00 & 0,90 & 0,29 & 0,44 & 0,57 & $-0,05$ & $-0,59$ \\
X-\% & 0,14 & 0,13 & 0,15 & 0,00 & 0,80 & 0,00 & 0,13 & 0,25 & 1,35 & 2,81 \\
Xu\% & 0,41 & 0,40 & 0,20 & 0,00 & 1,00 & 0,29 & 0,40 & 0,50 & 0,32 & 0,29 \\
S- & 0,17 & 0,00 & 0,40 & 0,00 & 2,00 & 0,00 & 0,00 & 0,00 & 2,14 & 3,78 \\
Zf & 2,58 & 2,00 & 1,81 & 0,00 & 8,00 & 1,00 & 2,00 & 4,00 & 0,58 & $-0,33$ \\
Ego & 2,94 & 3,00 & 1,62 & 0,00 & 7,00 & 2,00 & 3,00 & 4,00 & 0,24 & 0,21 \\
Ego/R & 0,41 & 0,43 & 0,21 & 0,00 & 1,50 & 0,33 & 0,43 & 0,56 & 0,60 & 4,37 \\
\hline
\end{tabular}

Tabela 4

Estatísticas Descritivas de 128 Crianças de 10 a 12 Anos do G2 no ZSC

\begin{tabular}{|c|c|c|c|c|c|c|c|c|c|c|}
\hline Variável & $M$ & $\mathrm{Me}$ & $D P$ & Min & Max & Per 25 & Per 50 & Per 75 & Assim & Kurt \\
\hline $\mathrm{R}$ & 7,15 & 7,00 & 1,87 & 3,00 & 15,0 & 6,00 & 7,00 & 8,00 & 1,27 & 2,45 \\
\hline W & 1,23 & 1,00 & 1,25 & 0,00 & 6,00 & 0,00 & 1,00 & 2,00 & 1,08 & 0,87 \\
\hline D & 4,45 & 5,00 & 1,78 & 0,00 & 8,00 & 3,00 & 5,00 & 5,75 & $-0,05$ & $-0,00$ \\
\hline $\mathrm{Dd}$ & 1,46 & 1,00 & 1,56 & 0,00 & 6,00 & 0,00 & 1,00 & 2,00 & 1,07 & 0,24 \\
\hline S & 0,98 & 1,00 & 0,97 & 0,00 & 6,00 & 0,00 & 1,00 & 2,00 & 1,33 & 4,16 \\
\hline $\mathrm{DQ}+$ & 4,31 & 4,00 & 2,21 & 0,00 & 13,0 & 3,00 & 4,00 & 6,00 & 0,34 & 0,95 \\
\hline DQo & 6,02 & 6,00 & 2,02 & 1,00 & 13,0 & 5,00 & 6,00 & 7,00 & 0,63 & 1,43 \\
\hline DQv/+ & 0,01 & 0,00 & 0,08 & 0,00 & 1,00 & 0,00 & 0,00 & 0,00 & 11,3 & 128 \\
\hline DQv & 0,70 & 0,00 & 0,28 & 0,00 & 2,00 & 0,00 & 0,00 & 0,00 & 4,37 & 20,5 \\
\hline $\mathrm{F}$ & 4,55 & 5,00 & 2,08 & 0,00 & 13,0 & 3,00 & 5,00 & 6,00 & 0,37 & 1,31 \\
\hline M & 0,63 & 0,00 & 0,85 & 0,00 & 4,00 & 0,00 & 0,00 & 1,00 & 1,35 & 1,55 \\
\hline FM & 0,36 & 0,00 & 0,69 & 0,00 & 4,00 & 0,00 & 0,00 & 1,00 & 2,37 & 6,82 \\
\hline M & 0,16 & 0,00 & 0,43 & 0,00 & 2,00 & 0,00 & 0,00 & 0,00 & 2,68 & 6,88 \\
\hline Ativo & 0,62 & 0,00 & 0,90 & 0,00 & 5,00 & 0,00 & 0,00 & 1,00 & 1,77 & 4,16 \\
\hline Passivo & 0,55 & 0,00 & 0,88 & 0,00 & 5,00 & 0,00 & 0,00 & 1,00 & 2,04 & 5,21 \\
\hline FC & 0,52 & 0,00 & 0,75 & 0,00 & 3,00 & 0,00 & 0,00 & 1,00 & 1,40 & 1,43 \\
\hline CF & 0,41 & 0,00 & 0,69 & 0,00 & 3,00 & 0,00 & 0,00 & 1,00 & 1,86 & 3,42 \\
\hline C & 0,07 & 0,00 & 0,33 & 0,00 & 3,00 & 0,00 & 0,00 & 0,00 & 6,29 & 47,3 \\
\hline FD & 0,09 & 0,00 & 0,30 & 0,00 & 2,00 & 0,00 & 0,00 & 0,00 & 3,77 & 14,9 \\
\hline SumC' & 0,48 & 0,00 & 1,05 & 0,00 & 9,00 & 0,00 & 0,00 & 1,00 & 4,91 & 34,6 \\
\hline SumV & 0,09 & 0,00 & 0,29 & 0,00 & 1,00 & 0,00 & 0,00 & 0,00 & 2,82 & 6,05 \\
\hline SumT & 0,05 & 0,00 & 0,21 & 0,00 & 1,00 & 0,00 & 0,00 & 0,00 & 4,33 & 17,0 \\
\hline SumY & 0,39 & 0,00 & 0,67 & 0,00 & 3,00 & 0,00 & 0,00 & 1,00 & 1,78 & 2,86 \\
\hline $\mathrm{Fr}+\mathrm{rF}$ & 0,04 & 0,00 & 0,19 & 0,00 & 1,00 & 0,00 & 0,00 & 0,00 & 4,81 & 21,5 \\
\hline GHR & 1,05 & 1,00 & 1,07 & 0,00 & 4,00 & 0,00 & 1,00 & 2,00 & 0,88 & 0,15 \\
\hline PHR & 0,81 & 1,00 & 0,99 & 0,00 & 5,00 & 0,00 & 1,00 & 1,00 & 1,65 & 3,35 \\
\hline (2) & 2,57 & 2,00 & 1,54 & 0,00 & 7,00 & 2,00 & 2,00 & 3,75 & 0,43 & $-0,01$ \\
\hline FQ+ & 0,00 & 0,00 & 0,00 & 0,00 & 0,00 & 0,00 & 0,00 & 0,00 & - & - \\
\hline FQo & 3,13 & 3,00 & 1,47 & 0,00 & 8,00 & 2,00 & 3,00 & 4,00 & 0,40 & 0,27 \\
\hline FQu & 2,95 & 3,00 & 1,92 & 0,00 & 11,0 & 1,25 & 3,00 & 4,00 & 1,09 & 1,99 \\
\hline FQ- & 0,99 & 1,00 & 1,08 & 0,00 & 5,00 & 0,00 & 1,00 & 1,75 & 1,33 & 2,10 \\
\hline
\end{tabular}


Tabela 4 (continuação)

Estatísticas Descritivas de 128 Crianças de 10 a 12 Anos do G2 no ZSC

\begin{tabular}{|c|c|c|c|c|c|c|c|c|c|c|}
\hline Variável & $M$ & $\mathrm{Me}$ & $D P$ & Min & $\operatorname{Max}$ & Per 25 & Per 50 & Per 75 & Assim & Kurt \\
\hline FQnone & 0,07 & 0,00 & 0,33 & 0,00 & 3,00 & 0,00 & 0,00 & 0,00 & 6,29 & 47,3 \\
\hline $\mathrm{H}$ & 0,82 & 1,00 & 0,88 & 0,00 & 4,00 & 0,00 & 1,00 & 1,00 & 0,99 & 1,02 \\
\hline $\mathrm{H}+(\mathrm{H})+\mathrm{Hd}+(\mathrm{Hd})$ & 1,78 & 2,00 & 1,45 & 0,00 & 7,00 & 1,00 & 2,00 & 3,00 & 1,03 & 1,40 \\
\hline A & 3,37 & 3,00 & 1,46 & 1,00 & 8,00 & 2,00 & 3,00 & 4,00 & 0,61 & 0,16 \\
\hline$A+(A)+A d+(A d)$ & 3,83 & 4,00 & 1,70 & 0,00 & 10,0 & 3,00 & 4,00 & 5,00 & 0,86 & 1,21 \\
\hline Art & 0,06 & 0,00 & 0,27 & 0,00 & 2,00 & 0,00 & 0,00 & 0,00 & 4,76 & 24,5 \\
\hline Ay & 0,08 & 0,00 & 0,26 & 0,00 & 1,00 & 0,00 & 0,00 & 0,00 & 3,18 & 8,25 \\
\hline $\mathrm{Na}$ & 0,23 & 0,00 & 0,47 & 0,00 & 2,00 & 0,00 & 0,00 & 0,00 & 1,90 & 2,91 \\
\hline $\mathrm{Cl}$ & 0,00 & 0,00 & 0,00 & 0,00 & 0,00 & 0,00 & 0,00 & 0,00 & - & - \\
\hline $\mathrm{Bt}$ & 0,61 & 0,00 & 0,84 & 0,00 & 5,00 & 0,00 & 0,00 & 1,00 & 1,80 & 5,06 \\
\hline Ls & 0,10 & 0,00 & 0,32 & 0,00 & 2,00 & 0,00 & 0,00 & 0,00 & 3,32 & 11,2 \\
\hline Geo & 0,00 & 0,00 & 0,00 & 0,00 & 0,00 & 0,00 & 0,00 & 0,00 & - & - \\
\hline Food & 0,07 & 0,00 & 0,28 & 0,00 & 2,00 & 0,00 & 0,00 & 0,00 & 4,37 & 20,5 \\
\hline $\mathrm{Bl}$ & 0,05 & 0,00 & 0,22 & 0,00 & 1,00 & 0,00 & 0,00 & 0,00 & 3,96 & 13,9 \\
\hline Sx & 0,00 & 0,00 & 0,00 & 0,00 & 0,00 & 0,00 & 0,00 & 0,00 & - & - \\
\hline $\mathrm{Fi}$ & 0,09 & 0,00 & 0,31 & 0,00 & 2,00 & 0,00 & 0,00 & 0,00 & 3,53 & 12,9 \\
\hline Ex & 0,00 & 0,00 & 0,00 & 0,00 & 0,00 & 0,00 & 0,00 & 0,00 & - & - \\
\hline $\mathrm{Cg}$ & 0,35 & 0,00 & 0,64 & 0,00 & 3,00 & 0,00 & 0,00 & 1,00 & 1,81 & 2,64 \\
\hline $\mathrm{P}$ & 1,14 & 1,00 & 1,06 & 0,00 & 5,00 & 0,00 & 1,00 & 2,00 & 0,95 & 0,73 \\
\hline DV & 0,11 & 0,00 & 0,33 & 0,00 & 2,00 & 0,00 & 0,00 & 0,00 & 3,13 & 9,86 \\
\hline INC & 0,13 & 0,00 & 0,39 & 0,00 & 2,00 & 0,00 & 0,00 & 0,00 & 3,36 & 11,2 \\
\hline DR & 0,10 & 0,00 & 0,49 & 0,00 & 5,00 & 0,00 & 0,00 & 0,00 & 7,91 & 74,0 \\
\hline FAB & 0,09 & 0,00 & 0,36 & 0,00 & 3,00 & 0,00 & 0,00 & 0,00 & 5,14 & 33,1 \\
\hline ALOG & 0,01 & 0,00 & 0,08 & 0,00 & 1,00 & 0,00 & 0,00 & 0,00 & 11,3 & 128 \\
\hline CONT & 0,00 & 0,00 & 0,00 & 0,00 & 0,00 & 0,00 & 0,00 & 0,00 & - & - \\
\hline Sum6 & 0,44 & 0,00 & 0,94 & 0,00 & 7,00 & 0,00 & 0,00 & 1,00 & 3,67 & 19,2 \\
\hline WSum6 & 1,06 & 0,00 & 2,61 & 0,00 & 21,0 & 0,00 & 0,00 & 1,00 & 4,58 & 28,4 \\
\hline WSumC & 0,77 & 0,50 & 0,91 & 0,00 & 4,50 & 0,00 & 0,50 & 1,00 & 1,47 & 2,39 \\
\hline EA & 1,39 & 1,00 & 1,37 & 0,00 & 6,50 & 0,00 & 1,00 & 2,00 & 1,27 & 1,55 \\
\hline Es & 1,47 & 1,00 & 1,62 & 0,00 & 8,00 & 0,00 & 1,00 & 2,00 & 1,79 & 4,31 \\
\hline SumC'-WSumC & $-0,28$ & 0,00 & 1,06 & $-4,50$ & 2,50 & $-1,00$ & 0,00 & 0,00 & $-0,58$ & 2,07 \\
\hline Afr & 0,56 & 0,50 & 0,19 & 0,25 & 1,50 & 0,50 & 0,50 & 0,64 & 2,07 & 6,73 \\
\hline Blends & 0,52 & 0,00 & 0,94 & 0,00 & 6,00 & 0,00 & 0,00 & 1,00 & 2,74 & 10,2 \\
\hline AG & 0,13 & 0,00 & 0,40 & 0,00 & 2,00 & 0,00 & 0,00 & 0,00 & 3,20 & 10,1 \\
\hline $\mathrm{COP}$ & 0,19 & 0,00 & 0,43 & 0,00 & 2,00 & 0,00 & 0,00 & 0,00 & 2,20 & 4,26 \\
\hline MOR & 0,23 & 0,00 & 0,56 & 0,00 & 3,00 & 0,00 & 0,00 & 0,00 & 2,94 & 9,50 \\
\hline PER & 0,26 & 0,00 & 0,80 & 0,00 & 7,00 & 0,00 & 0,00 & 0,00 & 5,45 & 39,4 \\
\hline Isol & 1,17 & 1,00 & 1,36 & 0,00 & 6,00 & 0,00 & 1,00 & 2,00 & 1,10 & 0,72 \\
\hline Isol/R & 0,16 & 0,13 & 0,18 & 0,00 & 0,80 & 0,00 & 0,13 & 0,29 & 1,06 & 0,64 \\
\hline Intelec & 0,15 & 0,00 & 0,41 & 0,00 & 2,00 & 0,00 & 0,00 & 0,00 & 2,92 & 8,33 \\
\hline $\mathrm{XA} \%$ & 0,85 & 0,86 & 0,14 & 0,17 & 1,00 & 0,78 & 0,86 & 1,00 & $-1,28$ & 2,69 \\
\hline WDA\% & 0,97 & 1,00 & 0,10 & 0,00 & 1,00 & 1,00 & 1,00 & 1,00 & $-7,46$ & 65,0 \\
\hline$X+\%$ & 0,44 & 0,44 & 0,21 & 0,00 & 1,00 & 0,29 & 0,44 & 0,57 & 0,22 & $-0,37$ \\
\hline$X-\%$ & 0,13 & 0,13 & 0,14 & 0,00 & 0,67 & 0,00 & 0,13 & 0,20 & 1,08 & 1,08 \\
\hline $\mathrm{Xu} \%$ & 0,40 & 0,39 & 0,21 & 0,00 & 0,92 & 0,20 & 0,39 & 0,55 & 0,23 & $-0,59$ \\
\hline S- & 0,13 & 0,00 & 0,36 & 0,00 & 2,00 & 0,00 & 0,00 & 0,00 & 2,67 & 6,47 \\
\hline $\mathrm{Zf}$ & 2,27 & 2,00 & 1,67 & 0,00 & 7,00 & 1,00 & 2,00 & 3,00 & 0,67 & 0,04 \\
\hline Ego & 2,68 & 2,50 & 1,69 & 0,00 & 9,00 & 2,00 & 2,50 & 4,00 & 0,66 & 0,72 \\
\hline Ego/R & 0,37 & 0,33 & 0,22 & 0,00 & 1,00 & 0,25 & 0,33 & 0,50 & 0,44 & 0,27 \\
\hline
\end{tabular}


Tabela 5

Estatísticas Descritivas de 61 Adolescentes de 13 a 14 Anos do G2 no ZSC

\begin{tabular}{|c|c|c|c|c|c|c|c|c|c|c|}
\hline Variável & $M$ & $\mathrm{Me}$ & $D P$ & Min & $\operatorname{Max}$ & Per 25 & Per 50 & Per 75 & Assim & Kurt \\
\hline $\mathrm{R}$ & 7,69 & 7,00 & 2,17 & 5,00 & 14,0 & 6,00 & 7,00 & 9,00 & 1,35 & 1,12 \\
\hline W & 1,28 & 1,00 & 0,95 & 0,00 & 4,00 & 1,00 & 1,00 & 2,00 & 0,60 & 0,07 \\
\hline D & 4,92 & 5,00 & 1,95 & 0,00 & 11,0 & 4,00 & 5,00 & 6,00 & 0,84 & 2,17 \\
\hline $\mathrm{Dd}$ & 1,49 & 1,00 & 1,52 & 0,00 & 7,00 & 0,00 & 1,00 & 3,00 & 1,18 & 1,55 \\
\hline S & 0,93 & 1,00 & 0,96 & 0,00 & 3,00 & 0,00 & 1,00 & 2,00 & 0,59 & $-0,79$ \\
\hline $\mathrm{DQ}+$ & 4,74 & 5,00 & 2,31 & 0,00 & 11,0 & 3,00 & 5,00 & 6,00 & 0,36 & 0,52 \\
\hline DQo & 6,67 & 6,00 & 2,20 & 2,00 & 12,0 & 5,00 & 6,00 & 8,00 & 0,32 & 0,20 \\
\hline DQv/+ & 0,00 & 0,00 & 0,00 & 0,00 & 0,00 & 0,00 & 0,00 & 0,00 & - & - \\
\hline DQv & 0,00 & 0,00 & 0,00 & 0,00 & 0,00 & 0,00 & 0,00 & 0,00 & - & - \\
\hline F & 4,90 & 5,00 & 2,07 & 1,00 & 11,0 & 3,00 & 5,00 & 6,00 & 0,84 & 0,71 \\
\hline M & 0,77 & 0,00 & 0,97 & 0,00 & 4,00 & 0,00 & 0,00 & 1,00 & 1,27 & 1,20 \\
\hline FM & 0,49 & 0,00 & 0,67 & 0,00 & 2,00 & 0,00 & 0,00 & 1,00 & 1,04 & $-0,08$ \\
\hline M & 0,13 & 0,00 & 0,38 & 0,00 & 2,00 & 0,00 & 0,00 & 0,00 & 3,09 & 9,78 \\
\hline Ativo & 0,92 & 1,00 & 1,11 & 0,00 & 4,00 & 0,00 & 1,00 & 1,50 & 1,13 & 0,49 \\
\hline Passivo & 0,48 & 0,00 & 0,67 & 0,00 & 2,00 & 0,00 & 0,00 & 1,00 & 1,10 & 0,03 \\
\hline FC & 0,54 & 0,00 & 0,74 & 0,00 & 3,00 & 0,00 & 0,00 & 1,00 & 1,25 & 0,92 \\
\hline $\mathrm{CF}$ & 0,43 & 0,00 & 0,69 & 0,00 & 3,00 & 0,00 & 0,00 & 1,00 & 1,66 & 2,50 \\
\hline C & 0,07 & 0,00 & 0,30 & 0,00 & 2,00 & 0,00 & 0,00 & 0,00 & 5,15 & 28,1 \\
\hline FD & 0,08 & 0,00 & 0,27 & 0,00 & 1,00 & 0,00 & 0,00 & 0,00 & 3,12 & 8,02 \\
\hline SumC' & 0,31 & 0,00 & 0,72 & 0,00 & 3,00 & 0,00 & 0,00 & 0,00 & 2,50 & 5,83 \\
\hline SumV & 0,11 & 0,00 & 0,37 & 0,00 & 2,00 & 0,00 & 0,00 & 0,00 & 3,44 & 12,3 \\
\hline SumT & 0,08 & 0,00 & 0,33 & 0,00 & 2,00 & 0,00 & 0,00 & 0,00 & 4,42 & 20,7 \\
\hline SumY & 0,38 & 0,00 & 0,82 & 0,00 & 5,00 & 0,00 & 0,00 & 1,00 & 3,50 & 16,4 \\
\hline $\mathrm{Fr}+\mathrm{rF}$ & 0,02 & 0,00 & 0,12 & 0,00 & 1,00 & 0,00 & 0,00 & 0,00 & 7,81 & 61,0 \\
\hline GHR & 1,23 & 1,00 & 1,03 & 0,00 & 4,00 & 0,50 & 1,00 & 2,00 & 0,80 & 0,24 \\
\hline PHR & 0,77 & 1,00 & 0,88 & 0,00 & 3,00 & 0,00 & 1,00 & 1,00 & 0,92 & 0,02 \\
\hline (2) & 2,72 & 3,00 & 1,68 & 0,00 & 9,00 & 2,00 & 3,00 & 3,00 & 1,38 & 3,23 \\
\hline $\mathrm{FQ}+$ & 0,02 & 0,00 & 0,12 & 0,00 & 1,00 & 0,00 & 0,00 & 0,00 & 7,81 & 61,0 \\
\hline FQo & 3,59 & 3,00 & 1,52 & 1,00 & 8,00 & 3,00 & 3,00 & 4,00 & 0,49 & 0,33 \\
\hline FQu & 3,10 & 3,00 & 1,85 & 0,00 & 9,00 & 2,00 & 3,00 & 4,00 & 0,60 & 0,55 \\
\hline FQ- & 0,97 & 1,00 & 0,99 & 0,00 & 4,00 & 0,00 & 1,00 & 2,00 & 0,89 & 0,28 \\
\hline FQnone & 0,00 & 0,00 & 0,00 & 0,00 & 0,00 & 0,00 & 0,00 & 0,00 & - & - \\
\hline $\mathrm{H}$ & 1,15 & 1,00 & 0,99 & 0,00 & 4,00 & 0,00 & 1,00 & 2,00 & 0,84 & 0,65 \\
\hline $\mathrm{H}+(\mathrm{H})+\mathrm{Hd}+(\mathrm{Hd})$ & 1,84 & 2,00 & 1,38 & 0,00 & 6,00 & 1,00 & 2,00 & 2,50 & 1,13 & 1,62 \\
\hline A & 3,64 & 3,00 & 1,77 & 0,00 & 8,00 & 2,00 & 3,00 & 5,00 & 0,42 & $-0,58$ \\
\hline $\mathrm{A}+(\mathrm{A})+\mathrm{Ad}+(\mathrm{Ad})$ & 4,13 & 4,00 & 1,83 & 1,00 & 9,00 & 3,00 & 4,00 & 5,00 & 0,49 & $-0,19$ \\
\hline Art & 0,05 & 0,00 & 0,21 & 0,00 & 1,00 & 0,00 & 0,00 & 0,00 & 4,27 & 16,8 \\
\hline Ay & 0,07 & 0,00 & 0,25 & 0,00 & 1,00 & 0,00 & 0,00 & 0,00 & 3,59 & 11,3 \\
\hline $\mathrm{Na}$ & 0,21 & 0,00 & 0,48 & 0,00 & 2,00 & 0,00 & 0,00 & 0,00 & 2,28 & 4,68 \\
\hline $\mathrm{Cl}$ & 0,00 & 0,00 & 0,00 & 0,00 & 0,00 & 0,00 & 0,00 & 0,00 & - & - \\
\hline $\mathrm{Bt}$ & 0,84 & 1,00 & 0,86 & 0,00 & 4,00 & 0,00 & 1,00 & 1,00 & 1,14 & 1,91 \\
\hline Ls & 0,15 & 0,00 & 0,35 & 0,00 & 1,00 & 0,00 & 0,00 & 0,00 & 2,03 & 2,22 \\
\hline Geo & 0,00 & 0,00 & 0,00 & 0,00 & 0,00 & 0,00 & 0,00 & 0,00 & - & - \\
\hline Food & 0,03 & 0,00 & 0,18 & 0,00 & 1,00 & 0,00 & 0,00 & 0,00 & 5,38 & 27,8 \\
\hline $\mathrm{Bl}$ & 0,03 & 0,00 & 0,25 & 0,00 & 2,00 & 0,00 & 0,00 & 0,00 & 7,81 & 61,0 \\
\hline Sx & 0,02 & 0,00 & 0,12 & 0,00 & 1,00 & 0,00 & 0,00 & 0,00 & 7,81 & 61,0 \\
\hline $\mathrm{Fi}$ & 0,16 & 0,00 & 0,45 & 0,00 & 2,00 & 0,00 & 0,00 & 0,00 & 2,88 & 7,99 \\
\hline Ex & 0,00 & 0,00 & 0,00 & 0,00 & 0,00 & 0,00 & 0,00 & 0,00 & - & - \\
\hline $\mathrm{Cg}$ & 0,18 & 0,00 & 0,46 & 0,00 & 2,00 & 0,00 & 0,00 & 1,00 & 2,66 & 6,68 \\
\hline $\mathrm{P}$ & 1,52 & 1,00 & 1,13 & 0,00 & 5,00 & 1,00 & 1,00 & 2,00 & 0,82 & 1,20 \\
\hline
\end{tabular}


Tabela 5 (continuação)

Estatísticas Descritivas de 61 Adolescentes de 13 a 14 Anos do G2 no ZSC

\begin{tabular}{|c|c|c|c|c|c|c|c|c|c|c|}
\hline Variável & $M$ & $\mathrm{Me}$ & $\mathrm{DP}$ & Min & Max & Per 25 & Per 50 & Per 75 & Assim & Kurt \\
\hline DV & 0,15 & 0,00 & 0,44 & 0,00 & 2,00 & 0,00 & 0,00 & 0,00 & 3,14 & 9,60 \\
\hline INC & 0,08 & 0,00 & 0,33 & 0,00 & 2,00 & 0,00 & 0,00 & 0,00 & 4,42 & 20,7 \\
\hline $\mathrm{DR}$ & 0,08 & 0,00 & 0,33 & 0,00 & 2,00 & 0,00 & 0,00 & 0,00 & 4,42 & 20,7 \\
\hline FAB & 0,00 & 0,00 & 0,00 & 0,00 & 0,00 & 0,00 & 0,00 & 0,00 & - & - \\
\hline ALOG & 0,00 & 0,00 & 0,00 & 0,00 & 0,00 & 0,00 & 0,00 & 0,00 & - & - \\
\hline CONT & 0,00 & 0,00 & 0,00 & 0,00 & 0,00 & 0,00 & 0,00 & 0,00 & - & - \\
\hline Sum6 & 0,31 & 0,00 & 0,62 & 0,00 & 2,00 & 0,00 & 0,00 & 1,00 & 1,84 & 2,21 \\
\hline WSum6 & 0,55 & 0,00 & 1,27 & 0,00 & 6,00 & 0,00 & 0,00 & 1,00 & 2,70 & 7,41 \\
\hline WSumC & 0,79 & 0,50 & 0,85 & 0,00 & 3,00 & 0,00 & 0,50 & 1,50 & 0,94 & 0,04 \\
\hline EA & 1,56 & 1,50 & 1,18 & 0,00 & 4,50 & 0,75 & 1,50 & 2,50 & 0,45 & $-0,54$ \\
\hline Es & 1,51 & 1,00 & 1,68 & 0,00 & 10,0 & 0,00 & 1,00 & 2,00 & 2,40 & 9,57 \\
\hline SumC'-WSumC & $-0,51$ & $-0,50$ & 1,08 & $-3,00$ & 3,00 & $-1,00$ & $-0,50$ & 0,00 & 0,59 & 2,09 \\
\hline Afr & 0,52 & 0,50 & 0,16 & 0,20 & 1,00 & 0,42 & 0,50 & 0,58 & 1,01 & 1,55 \\
\hline Blends & 0,56 & 0,00 & 0,82 & 0,00 & 3,00 & 0,00 & 0,00 & 1,00 & 1,36 & 1,00 \\
\hline AG & 0,26 & 0,00 & 0,57 & 0,00 & 3,00 & 0,00 & 0,00 & 0,00 & 2,66 & 8,47 \\
\hline $\mathrm{COP}$ & 0,20 & 0,00 & 0,47 & 0,00 & 2,00 & 0,00 & 0,00 & 0,00 & 2,46 & 5,59 \\
\hline MOR & 0,20 & 0,00 & 0,51 & 0,00 & 2,00 & 0,00 & 0,00 & 0,00 & 2,62 & 6,14 \\
\hline PER & 0,08 & 0,00 & 0,27 & 0,00 & 1,00 & 0,00 & 0,00 & 0,00 & 3,12 & 8,02 \\
\hline Isol & 1,40 & 1,00 & 1,39 & 0,00 & 5,00 & 0,00 & 1,00 & 2,00 & 1,01 & 0,22 \\
\hline Isol/R & 0,19 & 0,14 & 0,20 & 0,00 & 0,83 & 0,00 & 0,14 & 0,31 & 1,25 & 1,14 \\
\hline Intelec & 0,16 & 0,00 & 0,58 & 0,00 & 4,00 & 0,00 & 0,00 & 0,00 & 5,20 & 31,9 \\
\hline XA\% & 0,87 & 0,88 & 0,11 & 0,56 & 1,00 & 0,83 & 0,88 & 1,00 & $-0,60$ & $-0,4$ \\
\hline WDA\% & 0,99 & 1,00 & 0,76 & 0,40 & 1,00 & 1,00 & 1,00 & 1,00 & $-7,8$ & 61,0 \\
\hline$X+\%$ & 0,48 & 0,50 & 0,19 & 0,10 & 1,00 & 0,33 & 0,50 & 0,57 & 0,41 & 0,24 \\
\hline$X-\%$ & 0,12 & 0,13 & 0,11 & 0,00 & 0,44 & 0,00 & 0,13 & 0,17 & 0,62 & $-0,47$ \\
\hline $\mathrm{Xu} \%$ & 0,39 & 0,38 & 0,19 & 0,00 & 0,86 & 0,29 & 0,38 & 0,50 & 0,00 & $-0,13$ \\
\hline S- & 0,11 & 0,00 & 0,32 & 0,00 & 1,00 & 0,00 & 0,00 & 0,00 & 2,47 & 4,28 \\
\hline $\mathrm{Zf}$ & 2,44 & 2,00 & 1,47 & 0,00 & 6,00 & 1,00 & 2,00 & 3,00 & 0,56 & 0,10 \\
\hline Ego & 2,75 & 3,00 & 1,70 & 0,00 & 9,00 & 2,00 & 3,00 & 3,50 & 1,30 & 2,81 \\
\hline Ego/R & 0,35 & 0,33 & 0,17 & 0,00 & 0,71 & 0,22 & 0,33 & 0,50 & 0,07 & $-0,62$ \\
\hline
\end{tabular}

\section{Considerações Finais}

Diante dos dados encontrados, foram apresentadas tabelas que descrevem o desempenho médio de uma amostra de crianças e adolescentes no ZSC. Considerando o desempenho dessa amostra, pode-se perceber que, à medida que a idade aumenta, os processos cognitivos tornam-se mais eficazes. Os resultados permitem constatar que algumas variáveis relacionadas ao desempenho cognitivo foram mais sensíveis à dinâmica do desenvolvimento psicológico na infância, discriminando os três grupos etários e mostrando-se relacionadas com a idade cronológica. Nesse sentido, o ZSC revelou-se um método sensível para a avaliação de crianças e adolescentes não pacientes, considerando especialmente os aspectos cognitivos.

É importante salientar, ainda, que o presente estudo abarcou o desempenho de crianças e adolescentes de uma região que, até então, não tinha sido contemplada na amostra brasileira para o estudo do ZSC. Não obstante, sugere-se que mais estudos que abordem o desempenho de crianças no Zulliger sejam realizados em diferentes regiões do Brasil, a fim de ampliar o estudo do ZSC no país, abarcando todas as idades, as diferenças entre os sexos e a origem escolar da população infantojuvenil. A origem escolar é uma questão cuja investigação é imprescindível no caso de pesquisas futuras dedicadas à compreensão do desempenho de crianças e adolescentes. Isso deve permitir esclarecimentos sobre a forma como as condições de ensino de instituições públicas e privadas impactam sobre as características de personalidade do público analisado e, também, sobre a necessidade de serem construídas tabelas distintas em função dessa particularidade.

Outra sugestão para investigações futuras seria um maior investimento em estudos que abarcam populações de crianças com os mais diversos transtornos psicológicos. Essas investigações são importantes para identificar 
as variáveis que podem discriminar, de uma forma melhor, desempenhos pouco adaptativos e demonstrar de maneira eficaz a utilidade clínica do instrumento para alguns contextos mais específicos.

Contudo, a amostra estudada retrata o desempenho típico de jovens de 7 a 14 anos, especialmente por se tratar de crianças sem histórico recente de desordens psicológica ou psiquiátrica, sem reprovação até o ano da coleta de dados e com comprovada ausência de indícios de deficiência intelectual. Além disso, as crianças foram provenientes de 12 escolas distribuídas pelas sete principais regiões da cidade, além de outras quatro escolas do interior do estado. Por este motivo, os participantes podem ser considerados uma amostra adequada para os objetivos deste estudo. E, apesar do comportamento das variáveis ter respeitado tendências e observações da literatura científica, recomenda-se que outras pesquisas sejam implementadas para ampliação dos achados.

\section{Referências}

Associação Brasileira de Empresas de Pesquisa, ABEP (2014). Critério de Classificação Econômica Brasil. Recuperado de http://www.abep.org Angelini, A. L., Alves, I. C. B., Custódio, E. M., Duarte, W. F., \& Duarte, J. L. M. (1999). Matrizes Progressivas Coloridas de Raven: Escala Especial. Manual. São Paulo: CETEPP.

Biasi, F. C., \& Villemor-Amaral, A. E. (2016) Evidências de Validade do Zulliger-SC para Avaliação do Relacionamento Interpessoal de Crianças. Psico, 47(1), 13-23. doi:10.15448/1980-8623.2016.1.19990

Biasi, F. C. (2013). Evidências de Validade do Zulliger-SC para Avaliação do Relacionamento Interpessoal de Crianças (Dissertação de mestrado). Universidade São Francisco, Itatiba, SP.

Borges, A., Matos, M. G., \& Diniz, J. A. (2011). Processo Adolescente e Saúde Positiva: Âmbitos Afectivo e Cognitivo. Psicologia: Reflexão e Crítica, 24(2), 281-291. doi:10.1590/S0102-79722011000200009

Campagna, V. N., \& Souza, A. S. L. (2006). Corpo e Imagem Corporal no Início da Adolescência Feminina. Boletim de Psicologia, 56(124), 9-35. Recuperado de http://pepsic.bvsalud.org/pdf/bolpsi/v56n124/v56n124a03.pdf

Carretero, M., \& León, J. A. (2008). Do pensamento formal à mudança conceitual na adolescência. Em C. C. Marchesi \& A. Palácios, J. (Eds.). Desenvolvimento Psicológico e Educação. (pp. 323-334). Porto Alegre: Artmed.

Ceballos, G. (2009). El adolescente y sus retos: La aventura de hacerse mayor. Madrid: Pirámide.

Cohen, J. (1988). Statistical power analysis for the behavioral sciences. Hillsdale, NJ: Erlbaum.

Coll, C., Marchesi, A., \& Palácios, J. (Eds.). (2008). Desenvolvimento Psicológico e Educação. 2a edição. Porto Alegre: Artmed.

Eschenbeck, H., Kohlmann, C. W., \& Lohaus, A. (2007). Gender diferences in coping strategies in children and adolescents. Journal of Individual Differences, 28(1), 18-26. doi:10.1027/1614-0001.28.1.18

Exner, J. E. (2003). The Rorschach: A Compreensive System. (4a edição). New York: John Wiley \& Sons.

Exner, J. E., \& Sendín, C. (1999). Manual de Interpretação do Rorschach para o Sistema Compreensivo. São Paulo: Casa do Psicólogo.

Exner, J. E., \& Weiner, I. B. (1995). The Rorschach a comprehensive system: Assessment of children and adolescents second edition. (3a edição). New York: John Wiley \& Sons.

Fleiss, J. L. (1981). Métodos estatísticos para taxas e proporções. 2a edição. New York: John Wiley.

Hosseininasab, A., Mohammadi, M. R., Weiner, I. B., \& Delavar, A. (2015). Rorschach comprehensive system data for a sample of 478 iranian children at four ages. Journal of Personality Assessment, 97(2), 123-135. doi: 10.1080/00223891.2014.933345

Inhelder, B., Piaget, J. (1995). De la logique de l'enfant a la logique de l'adolescent. Paris: PUF.

Leichtman, M. (1996). The nature of the Rorschach task. Journal of Personality Assessment, 67(3), 478-493. doi: 10.1207/s15327752jpa6703_4

Martí, E. (2008). Processos cognitivos básicos e desenvolvimento intelectual entre os seis anos e a adolescência. Em C. C., Marchesi \& $\bar{A}$., Palácios, J. (Eds.). Desenvolvimento Psicológico e Educação. (pp. 233-251). Porto Alegre: Artmed.

Meyer, G. J, \& Kurtz, J. E. (2006). Advancing personality assessment terminology: Time to retire "objective" and "projective" as personalitytest descriptors. Journal of Personality Assessment, 87(3), 223-225. doi: 10.1207/s15327752jpa8703_01

Meyer, G. J., Hilsenroth, M. J., Baxter, D., Exner, J. E., Fowler, J. C., \& Piers, C. C., Resnick, J. (2002). An examination of interrater reliability for scoring the Rorschach Comprehensive System in eight data sets. Journal of Personality Assessment, 78(2), 219-274. doi: 10.1207/S15327752JPA7802_03

Meyer, G. J., Viglione, D., Mihura, J. L., Erard, R. E, Erdberg, P. (2017). R-PAS - Sistema de Avaliação por Performance no Rorschach. São Paulo: Hogrefe CETEPP.

Moreno, G. L. (2008). As Crianças e suas Infâncias: O brincar em diferentes contextos. Londrina: Humanidades.

Nascimento, R. S. G. F., Resende, A. C., Ribeiro, R. S. (2017). Crianças, Adolescentes e o Método de Rorschach. São Paulo: Pearson

Oliva, A. (2008). Desenvolvimento da personalidade durante a adolescência. Em C. C. Marchesi \& A. Palácios, J. (Eds.). Desenvolvimento Psicológico e Educação. (Vol. 1. 2 ed. pp 335-349). Porto Alegre: Artmed.

Pfundt, H., \& Duit, R. (1993). Bibliography. Students alternative frameworks and Science education. (4a edição). Germany: Institut für die Padagogikder Naturwissenschaften.

Poletto, M., \& Koller, S. H. (2011). Bem-estar subjetivo em crianças e adolescentes em situação de vulnerabilidade social. Psicologia: Reflexão e Crítica, 24(3), 476-484. doi:10.1590/S0102-79722011000300008

Raven, J. C. (2008). Teste das matrizes progressivas escala geral. Manual. Rio de Janeiro: Centro Editor de Psicologia Aplicada.

Resende, A. C., Carvalho, T. C.R., Martins, W. (2012). Desempenho Médio de Crianças e Adolescentes no Método de Rorschach Sistema Compreensivo. Avaliação Psicológica, 11(3), 375-394. Recuperado de http://pepsic.bvsalud.org/pdf/avp/v11n3/v11n3a06.pdf

Ribeiro, R. K. S. M., Semer, N. L., Yazigi, L. (2011). Rorschach Comprehensive System norms in Brazilian children from public and private schools. Psicologia: Reflexão e Crítica, 24(4), 671-684. doi: 10.1590/S0102-79722011000400007 
Silva, D. R., \& Campos, R. (2000). Some Rorschach variables from a normative study with 10-year-old Portuguese children. Rorschachiana, 24(1), 110-126. doi: 10.1027/1192-5604.24.1.110

Tavella, R. R., \& Villemor-Amaral, A. E. (2014). O Teste de Zulliger-SC: avaliação da criatividade em crianças. Estudos de Psicologia, 31(4), 489-497. doi: 10.1590/0103-166X2014000400003

Tavella, R. R. (2012). O Teste de Zulliger-SC: Avaliação da Criatividade em Crianças (Dissertação de mestrado). Universidade de São Francisco, Itatiba, SP.

Villemor-Amaral, A. E. d., Pavan, P. M. P., Tavella, R. R., Cardoso, L. M., \& Biasi, F. C. (2016). Validity evidence of the Z-Test-SC for use with children. Paidéia, 26(64), 199-206. doi: 10.1590/1982-43272664201607

Villemor-Amaral, A. E., \& Primi, R. (2012). O Teste de Zulliger no Sistema Compreensivo (ZSC): Forma individual. São Paulo: Casa do Psicólogo.

Villemor-Amaral, A. E., \& Vieira, P. G. (2016). Zulliger (CS) in Assesing the Relational Maturity of Children. Paidéia, 26(65), 369-376. doi: $10.1590 / 1982-43272665201601$

Wenar \& Curtis, 1991: Wenar, C., Curtis, K. M. (1991) The vality of the Rorschach for Assessing Cognitive and Affective Changes. Journal Personality Assessment, 57(2), 291-308. doi: 10.1207/s15327752jpa5702_8

Weiner, I. B., \& Craighead, W. E. (2010). The Corsini Encyclopedia of Psychology. New York: John Wiley and Sons.

\section{Sobre os autores}

Ana Clara Mateus Carvalho é psicóloga, Mestre pela Pontifícia Universidade Católica de Goiás.

Ana Cristina Resende é psicóloga, Doutora pela PUC RS e Pós-Doutora pela UNIFESP. Atualmente é professora ajunto de graduação e pós-graduação stricto sensu na PUC Goiás. 